\title{
Bursts of the Crab Nebula gamma-ray emission at high and ultra-high energies
}

\author{
A.S. Lidvansky ${ }^{\mathrm{a}}$ \\ Institute for Nuclear Research, Russian Academy of Sciences, Moscow, Russia
}

\begin{abstract}
Characteristics of the flares of gamma rays detected from the Crab Nebula by the AGILE and Fermi-LAT satellite instruments are compared with those of a gamma ray burst recorded by several air shower arrays on February 23, 1989 and with one recent observation made by the ARGO-YBJ array. It is demonstrated that though pulsar-periodicity and energy spectra of emissions at $100 \mathrm{MeV}$ (satellite gamma ray telescopes) and $100 \mathrm{TeV}$ (EAS arrays) are different, their time structures seem to be similar. Moreover, maybe the difference between "flares" and "waves" recently found in the Crab Nebula emission by the AGILE team also exists at ultra-high energies.
\end{abstract}

\section{Introduction}

The Gamma-ray flares from the Crab Nebula were discovered in a few-hundred-MeV energy range by the AGILE [1] and Fermi LAT [2] satellite telescopes. Since then, both telescopes continue recording such flares approximately once a year, the strongest of them (super-flare) having been detected in April 2011 [7,8]. The energy spectra of additional emission during the flares were measured to be different from those of the Nebula, however, "the mechanism driving the flares, their impulsive nature, the 12-month recurrence time, and the location, remain unknown" [12]. For all that, based on multi-wavelength campaign to study the Crab using Keck, the Hubble Space Telescope (HST), and the Chandra X-ray Observatory, the authors of [12] suggested the so-called "inner knot" to be just the emitting region for the flares. In addition, the analysis made by the AGILE collaboration for the September-October 2007 event has found a fine structure in the flare's time behavior [10]. They have demonstrated that there is a difference between shorter "flares" and longer "waves" in the Crab Nebula emission during this flare.

After the sensational discovery of gamma ray flares by AGILE and Fermi LAT it was recalled [3] that one event of this type (though at much higher energies) had been discovered many years ago by several EAS arrays [4-6].

\section{Gamma-Ray emission burst on February 23, 1989}

The first announcement about this burst was made during the International Workshop on Gamma-Ray Astronomy in the Crimea in 1989 [4] [4]. An increase in the intensity of extensive air showers (EAS) was detected by the Carpet air shower array of the Baksan Neutrino Observatory on February 23, 1989. After this communication, the group at Kolar Gold Fields (KGF) in India confirmed this result

a e-mail: lidvansk@lebedev.ru of Baksan and published a paper [5] on the simultaneous detection of a gamma-ray burst in the Crab Nebula at ultra high-energies independently by two EAS arrays. The final publication [6] by the Baksan and Durham University teams summarized the data of all arrays that could observe the source on this day. It was demonstrated that with different significance the burst was detected by all air shower arrays located in the longitude range from India to Italy (KGF, Tien Shan, Baksan and EAS TOP). The arrays located to the east and west of this interval (at that time OHYA MINE and Akeno SPICA in Japan and HEGRA at La Palma, Canary Islands, were in operation) showed no excess from the source direction. Thus, the total duration of the observed effect was no longer than about 7 hours. One can speak also about a possible decay of intensity whose maximum probably fell on observations with the KGF array. Figures 1 and 2 taken from paper [6] demonstrate the most remarkable features of the burst as detected by the Baksan Carpet air shower array.

It is clearly seen that the Baksan array data demonstrate an obvious dependence of the effect on the pulsar phase: events from a single phase bin can produce the entire observed excess of intensity (the KGF group also found some phase irregularity, though not so well pronounced). Summarizing, one can state that with a rather high probability (combined probability of random coincidences was estimated in [6] as $1.2510^{-7}$ ) a gamma-ray burst from the Crab Nebula was detected on February 23, 1989 in the energy range $10^{14}-10^{15} \mathrm{eV}$, and the excess emission in this burst is somehow connected with the pulsar's activity.

\section{Different and similar types of flares at different energies}

In [3] attention was drawn to the fact that the threepulse temporal structure in the event of February 23, 1989 might be reproduced in at least one of the AGILE flare events (September 2007). A bit later it has been discovered [10] that in addition to flares there exists 


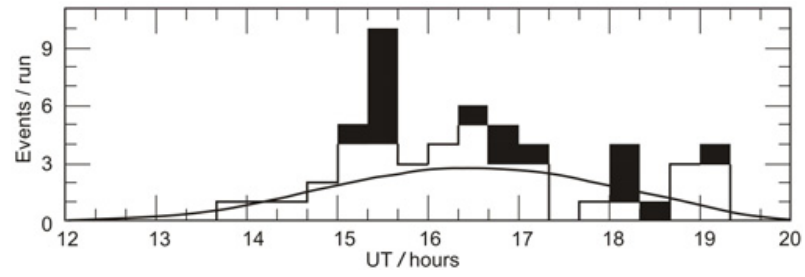

Figure 1. The number of events within the Crab cell per 20 min run for 23 February 1989. The smooth curve represents the expected background. Blacked events are from the 9th bin in Fig. 2.

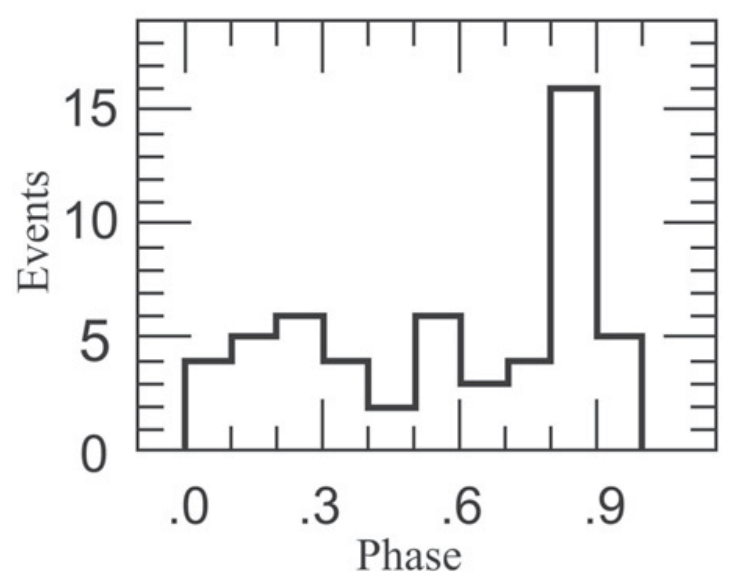

Figure 2. The relative phase distribution of the 55 events within the Crab cell after barycentring the arrival times and applying the values of the pulsar period and its derivative according to the Jodrell Bank Crab ephemeris.

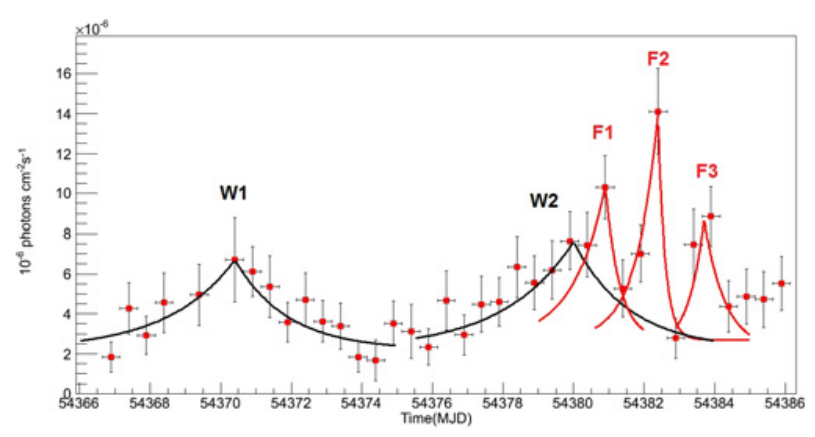

Figure 3. The difference between flares and waves in the Crab Nebula gamma-ray emission according to Striani et al. [10]. The three-flare structure of the event is similar to three bunches of narrow-phase emission constituting almost all excess counts in Fig. 1.

another type of intensity increase called waves. And exactly during September 2007 three short flares (F1, F2, and F3) were identified together with two waves W1 and W2 (see Fig. 3).

The similarity of the temporal structures for the events of Figs. 1 and 2 is obvious. In one case three bunches shorter than one hour are repeated with a period slightly longer than one hour. The other event includes three flares with duration of about one day, and they are repeated with a period a bit longer than one day. So, a sort of a stable pattern with a scale factor is observed at drastically different energies of gamma rays. It should be noted that the fine temporal structure was also reported by the Fermi LAT team for the March 2013 flare: "The light

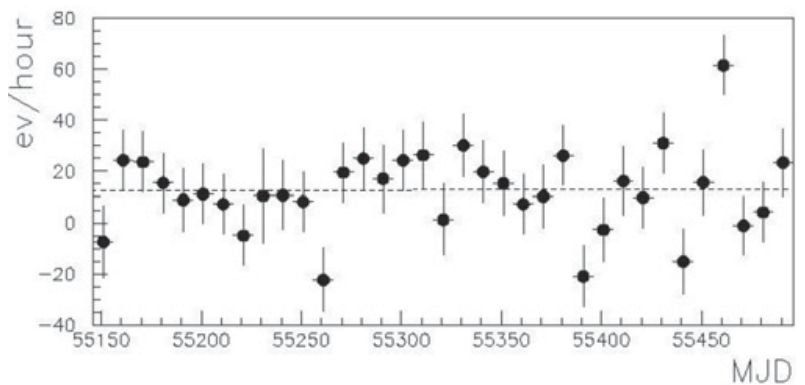

Figure 4. The Crab light curve obtained in September 2010 by the ARGO-YBJ array with 10-day bins. The flare recorded by satellite gamma-ray telescopes took place on September $8-22$. The maximum point is for September 19-26, 2010. Its significance is 5.4 standard deviations.

curve shows three sharp spikes (MJD 56357.1, 56357.9, 56360.1) on top of a strongly increased flux level." [15]. Here, the period between the spikes is not so constant, but its duration, nevertheless, is close to that of the Fig. 3 event.

The results of AGILE and Fermi LAT stimulated other groups to search for possible Crab flares, and one of them seemed, at first, to be successful. The ARGO-YBJ array after processing the data for the Crab direction has found [9] an enhancement for the period September 19-26, 2010, which is shown in Fig. 4 for a 10-day period of averaging. This result was obtained at energies (about $1 \mathrm{TeV}$ ) intermediate between the two regions considered above. Nevertheless, there is a temptation to think that the ratio of durations (several hours for the 1989 event and about a week in this case) gives some evidence in favor of the existence of the same "flares and waves" dichotomy at ultra-high energies. However, in the next publication [11] of ARGO-YBJ this piece of data was partially disavowed, though another interesting fact was presented: a correlation between Fermi and ARG-YBJ Crab intensities: "Even if the ARGO-YBJ rate variations are consistent with statistical fluctuations, the Fermi and ARGO-YBJ data seems to follow a similar trend. The ARGO-YBJ rate appears higher in the "hot" Fermi periods." [11].

It is commonly believed that the energy spectrum of the Crab Nebula is formed by synchrotron emission below $1 \mathrm{GeV}$ and by inverse Compton radiation at higher energies. As far as flaring radiation is concerned, it is presumed to be associated with the synchrotron component. Spectral behavior of the flares appeared to be rather different: according to Fermi LAT, the flare in February 2009 only exhibited a flux increase with no spectral change. On the contrary, the flares in September 2010 and April 2011 had fluxes strongly correlated with the spectral index. In the March 2013 flare "the spectrum of the synchrotron nebula hardens as the flux increases" [15]. At the same time, according to an analysis made in [15], within the measurement accuracy the spectrum of the pulsar did not change in the entire analysis window with respect to the all-time average, and only the synchrotron component of the Crab Nebula is rapidly variable.

At the moment no information on spectra is available for the ultra-high energy bursts.

The Crab pulsar emission has a very complicated energy dependence. In the radio waveband in addition to the main pulse (MP) and interpulse (IP) there are precursors of the MP, the low-frequency component (LFC) 
and two high-frequency components (HFC1 and HFC2), as well as giant radio pulses that randomly appear on different phases. At higher energies the Crab light curve becomes more regular and the gamma-ray domain has only two pulses P1 and P2. The amplitudes of these two pulses depend on energy. For example, the P2 pulse is twice larger in amplitude than P1 at an energy less than $10 \mathrm{MeV}$ (COMPTEL), but already at energies $>30 \mathrm{MeV}$ (EGRET) the situation is opposite. At energies exceeding $100 \mathrm{GeV}$ (the data of the VERITAS and MAGIC Cherenkov telescopes) pulse P2 becomes dominant again. There are also some indications to the possible appearance of a new pulse P3. So, it would be not surprising if at still higher energies only a single pulse survives, as it takes place in Fig. 2.

\section{Discussion and conclusions}

Interpretation of gamma-ray flares produced by synchrotron radiation of accelerated electrons in compact regions of an intensified magnetic field (plasma instabilities) requires the energy of such electrons to be as high as $10^{15} \mathrm{eV}$ (see for example, [10]). If these electrons do really exist, one can recall the idea of Atoyan and Aharonian [16] that Crab Nebula wisps probably have sufficient amount of matter to make bremsstrahlung radiation effective. If so, $1000 \mathrm{TeV}$ electrons can easily produce $100 \mathrm{TeV}$ gamma rays. Even without this hypothesis accelerated particles can simultaneously produce multi-TeV emission via inverse Compton effect and hundred-MeV - GeV emission through synchrotron radiation [17]. When explaining the two sets of data discussed in this paper, the problem is that at ultra-high energies pulsar periodicity takes place, while it is not observed in hundred-MeV flares. As a possible explanation one can suggest that the initial acceleration is directly connected with the pulsar, while at lower energies an observer receives gamma rays after randomization of the phase in the pulsar-wind driven shock.

Nevertheless, many details of the mechanisms of flare generation (like, for example, triple scaled temporal structure and distinction between flares and waves) are unclear as before, and the Crab Nebula still remains an enigmatic "astrophysical chimera" [13]. One can hope, however, that the above juxtaposition of data at different energies will be useful for construction of a realistic model of the source internal mechanism.

The work is partially supported by the Russian Foundation for Basic Research, grant no. 16-02-00687.

\section{References}

[1] M.Tavani et al., Science 331, 736-39 (2011)

[2] A.A. Abdo et al., Science 331, 739-42 (2011)

[3] A.S. Lidvansky, Journal of Physics: Conference Series 409, 012114 (2013)

[4] V.V. Alexeenko, A.S. Lidvansky and V.A. Tizengauzen V.A., Proc. of Int. Workshop on Very High Energy Gamma Ray Astronomy (Crimea, USSR), 17-21 April, 1989. Ed. by A.A. Stepanyan, D.J. Fegan and M.F. Cawley, p. 187

[5] B.S Acharya, M.V.S. Rao, K. Sivaprasad, B.V. Sreekantan and P.R. Vishwanath, Nature 347, 364-5 (1990).

[6] V.V. Alexeenko, Yu.M. Andreyev, A.E. Chudakov, Ya.S. Elensky, A.S. Lidvansky, S.Kh. Ozrokov, Yu.V. Stenkin, V.A. Tizengauzen, L.J. Graham, J.L. Osborne and A.W. Wolfendale, J. Phys. G: Nucl. Part. Phys. 18, L83-L88 (1992)

[7] R. Buehler et al., Astrophys. Journ. 749, 26 (2012)

[8] E. Striani et al., Astrophys. Journ. Lett. 741, L5 (2011)

[9] S. Vernetto et al., Proc. 32nd ICRC, Beijing, 2011

[10] E. Striani et al., Astrophys. Journ. 765, 52 (2013)

[11] B. Bartoli et al. Astrophys. Journ. 798, 119 (2015)

[12] A. Rudy et al., Astrophys. Journ. 811, 24 (2015)

[13] J.J. Hester, Annu. Rev. Astron. Astrophys. 46, 127-55 (2008)

[14] R. Bühler and R. Blandford, Rep. Prog. Phys. 77, 066901 (2014)

[15] M. Mayer et al., Astrophys. Journ. Lett. 775, L37 (2013)

[16] A.M. Atoyan and F.A. Aharonian, Mon. Not. Roy. Astron Soc. 278, 525 (1996)

[17] W. Bednarek and W. Idec, Mon. Not. Roy. Astron Soc. 414, 2229 (2011) 